\title{
Efficacy and safety of glucocorticoids in the treatment of community-acquired pneumonia: A meta-analysis of randomized controlled trials
}

\author{
Li-ping Chen ${ }^{1}$, Jun-hui Chen ${ }^{2}$, Ying Chen ${ }^{1}$, Chao Wu ${ }^{1}$, Xiao-hong Yang ${ }^{1}$ \\ ${ }^{1}$ Department of Respiratory and Critical Care Medicine, People's Hospital of Xinjiang Uygur Autonomous Region, \\ Urumqi 830001, China \\ ${ }^{2}$ Department of Pharmacy, Sixth Affiliated Hospital of Xinjiang Medical University, Urumqi 830002, China
}

Corresponding Author: Xiao-hong Yang,Email: clp651@126.com

BACKGROUND: Community-acquired pneumonia (CAP) is pneumonia acquired infectiously from normal social contact as opposed to being acquired during hospitalization. CAP is a leading cause of illness and death. This review aims to determine the efficacy and safety of glucocorticoids in the treatment of community-acquired pneumonia (CAP).

DATA SOURCES: We searched randomized controlled trials (RCTs) from Pubmed, EMBASE, Cochrane Library, Chinese Journal Full-text Database, and Chinese Biomedical Literature Database to obtain the information by using steroids, glucocorticoids, cortisol, corticosteroids, community-acquired pneumonia and CAP as key words. The quality of RCTs was evaluated. A Meta-analysis was made using RevMan 5.0 provided by the Cochrance Collaboration.

RESULTS: Seven RCTs involving 944 patients were included in the meta-analysis. The mean length of hospital stay in glucocorticoids treatment group was significantly shorter than that in standard treatment group $(W M D=-1.70,95 \% C l 2.01-1.39, Z=10.81, P<0.00001)$. No statistically significant differences were found in the mortality rate $(R R=0.77,95 \% \mathrm{Cl} 0.46-1.27, Z=1.03$, $P=0.30)$, the mean length of hospital stay in ICU $(W M D=1.17,95 \% C l 1.68-4.02, Z=0.81, P=0.42)$, the incidence of super infection $(R R=1.32,95 \% C l 0.66-2.63, Z=0.79, P=0.43)$, the incidence of hyperglycemia $(R R=1.84,95 \% C l 0.76-4.41, Z=1.36, P=0.17)$, the incidence of upper gastrointestinal bleeding $(R R=1.98,95 \% C l 0.37-10.59, Z=0.80, P=0.42)$ between the standard treatment group and the glucocorticoids treatment group.

CONCLUSIONS: The use of glucocorticoids in patients with community-acquired pneumonia can significantly shorten the duration of illness and have a favorable safety profile. However, it could not reduce the overall mortality.

KEY WORDS: Glucocorticoids; Community-acquired pneumonia; Meta-analysis

World J Emerg Med 2015;6(3):172-178

DOI: 10.5847/wjem.j.1920-8642.2015.03.002

\section{INTRODUCTION}

Community-acquired pneumonia (CAP) is pneumonia acquired infectiously from normal social contact as opposed to being acquired during hospitalization. Causes of CAP include bacteria, viruses, fungi, and parasites. CAP can be diagnosed simply by symptoms and physical examination or by X-rays, sputum test and others. CAP is a leading cause of illness and death. Its morbidity in the general population is $2 \%-12 \%$, whereas its mortality is up to $20 \%-50 \% .{ }^{[1]} \mathrm{CAP}$ is primarily treated with antibiotics. In recent years, treatments including administration of high-efficient broad spectrum antibiotics and mechanical ventilation have been widely used clinically, but there was no marked decrease of 
the CAP mortality. ${ }^{[2-4]}$ Therefore, researches of CAP treatment are turning to the subsidiary measure of nonantibiotics. Experiments have shown that the key factor for poor prognosis of CAP patient is the excessive immune-inflammatory reactions of the lungs and the whole body. Moreover, adrenal insufficiency has been diagnosed in gravis CAP patients, which is related to severity of the disease and its poor prognosis. ${ }^{[5,6]}$ Glucocorticoids (GCS) which have pronounced effects on inflammation and adrenal insufficiency remain to be the key adjuvant medicine. However, the use of GCS for the treatment of CAP remains debatable as it may disguise disease process to complicated diagnosis and delayed treatment, resulting in untoward reaction of GCS. Hence, this study aims to evaluate the relevant results from randomized controlled trials (RCTs) that have been published in recent years.

\section{METHODS \\ Inclusion criteria}

The included were patients aged more than 18 years old who had been diagnosed with CAP in accordance with China CAP Guidelines and the guidelines of the American Thoracic Society (ATS). The patients were treated at the emergency room or hospitalized.

\section{Exclusion criteria}

Clinical studies involved CAP patients with HIV and those with hospital acquired pneumonia were excluded. Studies with limited information, inaccurate number of cases, literature reviews, case reports, etc were also excluded.

\section{Interventions}

GCS combined with conventional antibiotic treatment or conventional antibiotic treatment only was given to the patients.

\section{Measurement index}

The mortality, lengh of hospital stay, lengh of ICU stay, occurrence of adverse reaction, including super infection, upper gastrointestinal bleeding (UGB), hyperglycemia were measured.

\section{Retrieval method}

A literature search was conducted with the following key words such as steroids, glucocorticoids, cortisol, corticosteroids, community-acquired pneumonia, CAP from Pubmed (January 1966 to December 2012), Embase (January 1974 to September 2012), and Cochrane Library $(2009,3 \mathrm{rd})$. These words were also searched from Wanfang Database (January 1982 to September 2012), Chinese Journal Full-text Database (January 1979 to September 2012) and Chinese Biomedical Study Database, and CBM (January 1978 to September 2012). Pubmed and Embase retrieval is limited to clinical studies. Searching language is limited to Chinese and English.

\section{Study screening and data extraction}

Two researchers separately screened the studies, collected and cross-checked data, and distinguished differences through discussion with another researcher. Missing materials were supplemented by contacting the authors through telephone or mails. Collected information mainly consisted of name of the authors, publication date, study resource, size of participating centers, type of the study design, sample size, description of intervention, location of diagnosis and treatment as well as observational indices.

\section{Quality evaluation}

Quality evaluation was done according to the quality evaluation standard recommended by Cochrane handbook 5.0: (1) whether the randomized method is correct or not; (2) whether allocation concealment method is used or not; (3) whether blinding or not is adopted; (4) whether there is bias caused by data deficient or not; (5) whether there is bias caused by selective report or not; (6) whether there are other types of biases or not. Each quality standard will be divided into "yes", "no" and "not clear".

\section{Statistical treatment}

Meta-analysis was made using RevMan 5.0 provided by the Cochrance Collaboration Center. Enumeration data evaluation adopted relative incidence (RR). When each document used the same measurement and unit to one index, measurement data counted weighted mean difference (WMD). Each effect size was expressed in $95 \%$ confidence interval (CI). The heterogeneity of each study was evaluated using the chi-square test. If there was no heterogeneity $\left(P<0.1, I^{2}>50 \%\right)$ between two studies, a fixed effect model was used for analysis; if there was heterogeneity $\left(P<0.1, I^{2}>50 \%\right)$, the data source and associated factors were analyzed. If there is statistical heterogeneity without clinical heterogeneity 
and statistical significance between studies, a random effect model was used for analysis. If the heterogeneity between the two groups was too large to search for the data source, descriptive analysis was used.

\section{RESULTS}

\section{Search results}

According to the designated searching method, 2471 relevant articles were retrieved in initial stage. Irrelevant articles which were eliminated after a review included essays, reviews, mails, meeting reports and repeated study reports. Thirteen articles were selected. After full-text review and in-depth information evaluation, the unstudied-researches, nonclinical study, medical interventions unrelated to GCS and studies with incomplete data were excluded. Final analysis included 7 articles that met the inclusion criteria.

\section{Quality evaluation and general characteristics of the included studies}

The quality evaluation of 7 articles is shown in Table 1 and their general characteristics are shown in Table 2. All articles reported the results from randomized studies, and random concealment was inadequate. In three studies $^{[8,10,11]}$ a single-blind method was used and a total of 944 patients were included.

\section{Meta-analysis \\ Improvement of mortality}

Six of the seven articles described the mortality of community-acquired pneumonia in both GCS treatment group and conventional treatment group, and they were subjected to meta-analysis. The heterogeneity $\left(P=0.47, I^{2}=0 \%\right)$ was not estimated using a fixedeffect mode. Compared to the conventional treatment group, the mortality of the GCS treatment group was reduced $(R R=0.77,95 \% C I 0.46-1.27)$, but the difference was not statistically significant $(Z=1.03$, $P=0.30$ ) (Figure 1).

\section{Mean length of hospital stay}

The mean length of hospital stay was described in six of the seven studies of CAP patients from both treatment groups. The heterogeneity $\left(P=0.04, I^{2}=56 \%\right)$ was estimated using a fixed-effect model. The mean length of hospital stay of CAP patients in the GCS treatment group was shorter $(W M D=-1.70,95 \% C I-2.01$ to -1.39$)$ than that in the conventional treatment group $(Z=10.81$, $P<0.01$ ) (Figure 2).

Because of the heterogeneity in the six studies, a random effect model was used to re-analyze the study results for further verification of the stability of metaanalysis. The results showed that the mean length of hospital stay of CAP patients from the GCS treatment

Table 1. The quality evaluation of the studies included

\begin{tabular}{|c|c|c|c|c|c|c|}
\hline References & $\begin{array}{l}\text { Correctness of } \\
\text { randomized studies }\end{array}$ & $\begin{array}{l}\text { Correctness of allocation } \\
\text { concealment methods }\end{array}$ & $\begin{array}{l}\text { Blinding method } \\
\text { taken or not }\end{array}$ & $\begin{array}{l}\text { Deviation caused } \\
\text { by data deficiency } \\
\text { or not }\end{array}$ & $\begin{array}{l}\text { Deviation caused } \\
\text { by selective reports } \\
\text { or not }\end{array}$ & $\begin{array}{l}\text { Deviation } \\
\text { caused by } \\
\text { others }\end{array}$ \\
\hline Snijders et $_{\text {al }}^{[7]}$ & Unclear & Unclear & Yes & No & No & No \\
\hline Remmelts et $\mathrm{al}^{[8]}$ & Unclear & Unclear & Yes & No & No & No \\
\hline Confalonieri et $\mathrm{al}^{[9]}$ & Yes & Yes & Yes & No & No & No \\
\hline Mikami et al ${ }^{[10]}$ & Unclear & Unclear & Yes & No & No & No \\
\hline Marik et al $^{[11]}$ & Yes & Unclear & Yes & No & No & No \\
\hline Meijvis et $\mathrm{al}^{[12]}$ & Yes & Yes & Yes & No & No & No \\
\hline Fernández-Serrano et al ${ }^{[13]}$ & Yes & Yes & Yes & No & No & No \\
\hline
\end{tabular}

Table 2. The general characteristics of the studies included

\begin{tabular}{|c|c|c|c|c|c|}
\hline References & Year & Country & Hospital scale & Research design & Total (Test/control) \\
\hline$\overline{\text { Snijders et } \mathrm{al}^{[7]}}$ & 2010 & Holland & A teaching hospital with 900 beds & Random control single center & $213(104 / 109)$ \\
\hline Remmelts et al ${ }^{[8]}$ & 2012 & Holland & $\begin{array}{l}\text { Three hospitals, } 1 \text { of them is } \\
\text { teaching hospital }\end{array}$ & Random control multicenter & $275(131 / 144)$ \\
\hline Confalonieri et al $^{[9]}$ & 2005 & Italy & Six hospitals & Random control multicenter & $46(23 / 23)$ \\
\hline Mikami et al ${ }^{[10]}$ & 2007 & Japan & General hospital in community & Random control single center & $31(15 / 16)$ \\
\hline Marik et al ${ }^{[11]}$ & 2008 & South Africa & $\begin{array}{l}\text { Multidisciplinary ICU tertiary care } \\
\text { teaching hospital }\end{array}$ & Random control single center & $30(14 / 16)$ \\
\hline Fernández-Serrano et $\mathrm{al}^{[13]}$ & 2011 & Spain & A hospital with 900 beds & Random control single center & $45(23 / 22)$ \\
\hline
\end{tabular}


group was shorter than that from the conventional treatment group $(W M D=-1.66,95 \% C I-2.37$ to -0.94$)$ $(Z=10.81, P<0.01)$.

\section{Mean length of hospital stay in ICU}

Three of the seven studies described the mean length of hospital stay in ICU for both groups. The heterogeneity $\left(P=0.07, I^{2}=62 \%\right)$ was estimated using a fixed-effect model. The mean length of hospital stay in ICU for the GCS treatment group was longer than that for the conventional treatment group $(W M D=1.17$, $95 \% C I-1.68$ to 4.02 ), but there was no significant difference ( $Z=0.81, P=0.42$ ) (Figure 3 ).
Because of the heterogeneity in the three studies, a random effect model was adopted to re-analyze for further verification of the stability of the result from meta-analysis. The mean length of hospital stay of CAP patients from the GCS treatment group was shorter than that from the conventional treatment group $(W M D=1.17$, $95 \% C I-4.39$ to 6.73 ), but there was no significant difference $(Z=0.41, P=0.68)$.

\section{Super infection}

Three of the seven studies described the incidence of super infection in both groups. The meta-analysis included these three studies. The heterogeneity $(P=0.11$,

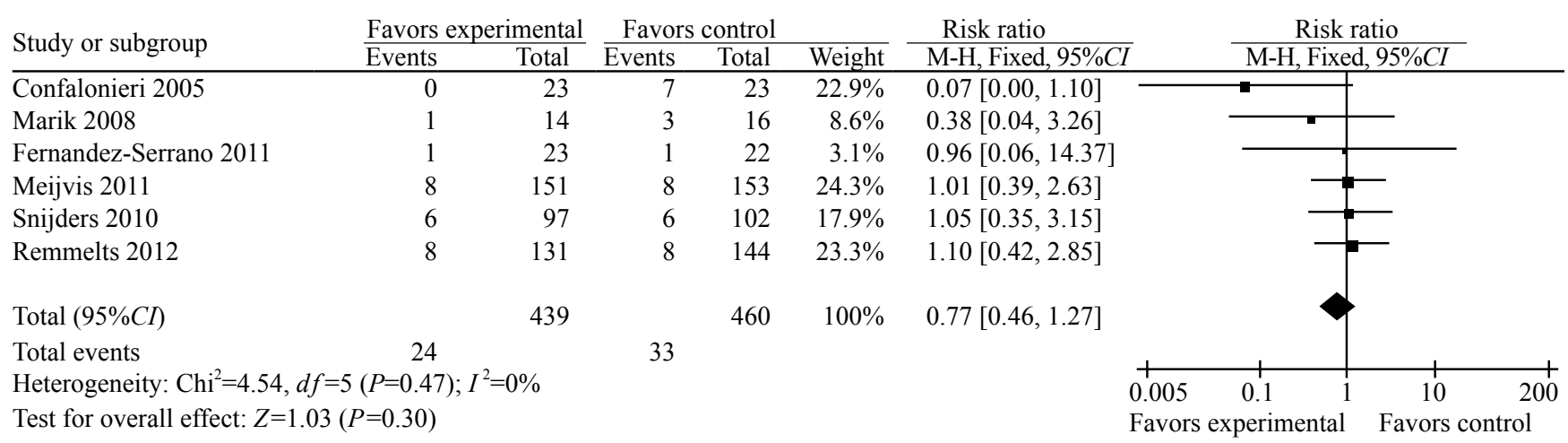

Figure 1. Comparison of mortality between the GCS treatment group and standard treatment group.

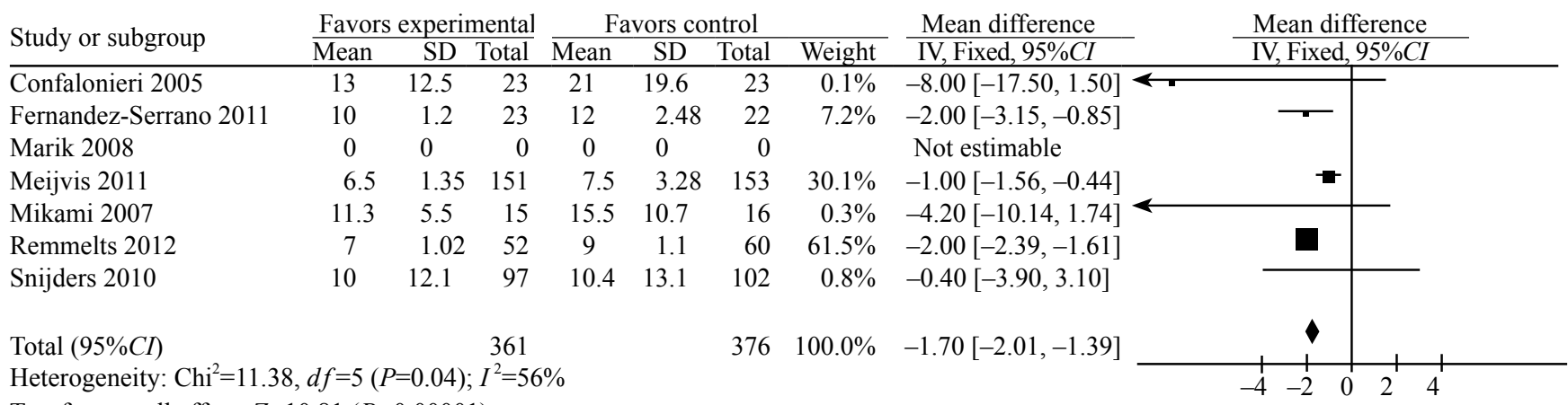

Test for overall effect: $Z=10.81(P=0.00001)$

Favors experimental Favors control

Figure 2. Comparison of the mean length of hospital stay in the two groups.

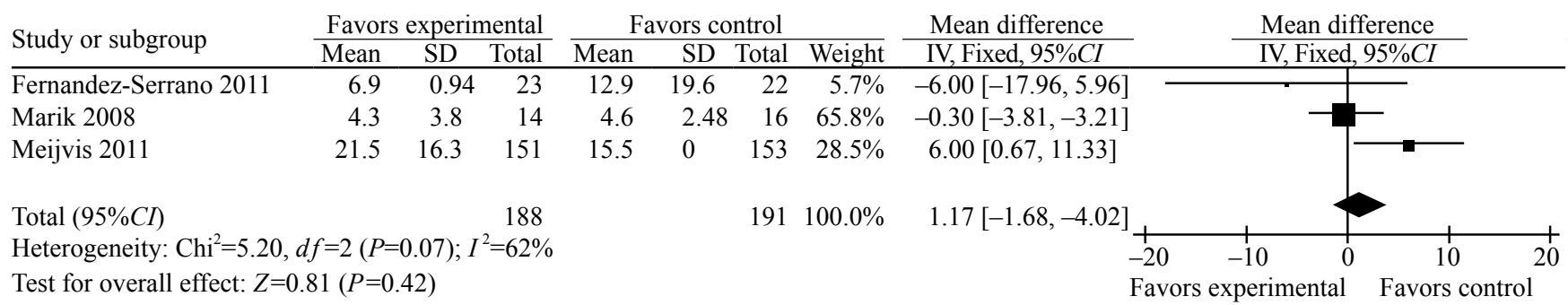

Figure 3. Comparison of the mean length of hospital stay at ICU in the two groups. 
$I^{2}=55 \%$ ) was estimated using a fixed-effect model. The incidence of super infection in the GCS treatment group was higher than that in the conventional treatment group ( $R R=1.32,95 \% C I 0.66$ to 2.63 ), but there was no significant difference $(Z=0.79, P=0.43)$ (Figure 4).

Owing to the heterogeneity in the 3 studies, a random effect model was adopted to re-analyze for further verification of the stability of the result from this meta-analysis. The incidence of super infection of CAP patients from the GCS treatment group was higher than that from the conventional treatment group $(R R=1.25$, $95 \% C I 0.35$ to 4.45$)$, but there was no significant difference $(Z=0.34, P=0.73)$.

\section{Hyperglycemia}

Three of the seven studies described the incidence of hyperglycemia (HG) in the two groups. The meta- analysis analyzed the data from the 3 studies. The heterogeneity $\left(P=0.79, I^{2}=0\right)$ was not estimated using a fixed-effect model. The incidence of HG in the GCS treatment group was higher than that in the conventional treatment group ( $R R=1.84,95 \% C I 0.76$ to 4.41$)$, but the difference was not statistically significant $(Z=1.36$, $P=0.17$ ) (Figure 5).

\section{Upper gastrointestinal bleeding}

Three of the 7 studies described the incidence of upper gastrointestinal bleeding in both treatment groups. The meta-analysis included the 3 studies. And the heterogeneity $\left(P=0.83, I^{2}=0 \%\right)$ was not estimated using a fixed-effect model. The incidence of upper gastrointestinal bleeding in the GCS treatment group was higher than that in the conventional treatment group ( $R R=1.98,95 \% C I 0.37$ to 10.59 ), but there was

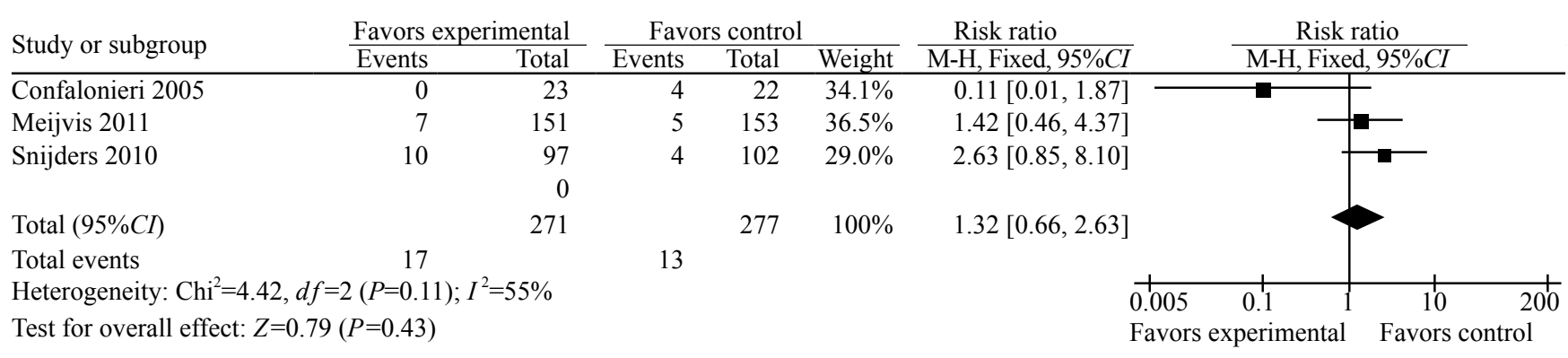

Figure 4. Comparison of the incidence of super infection in the two groups.

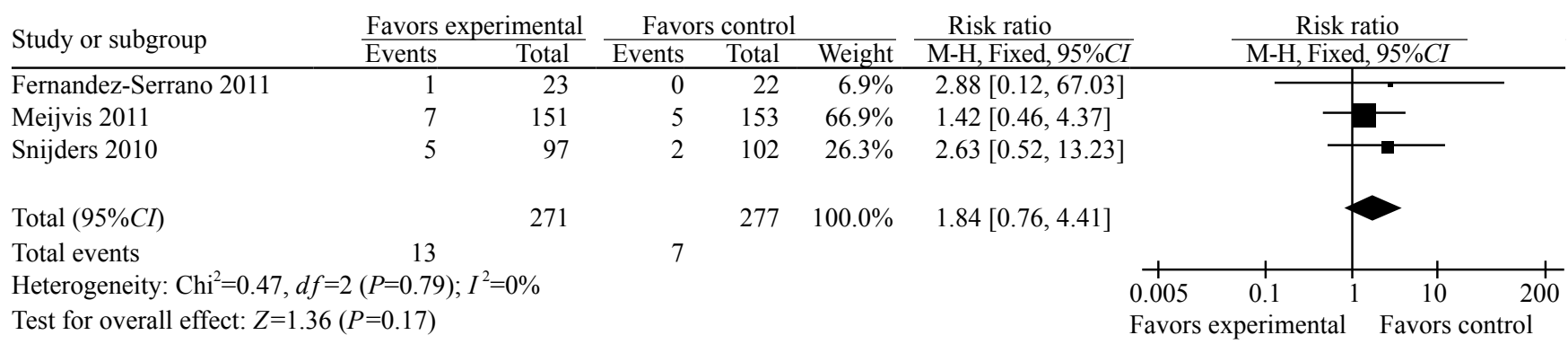

Figure 5. Comparison of the incidence of hyperglycemia in the two groups.

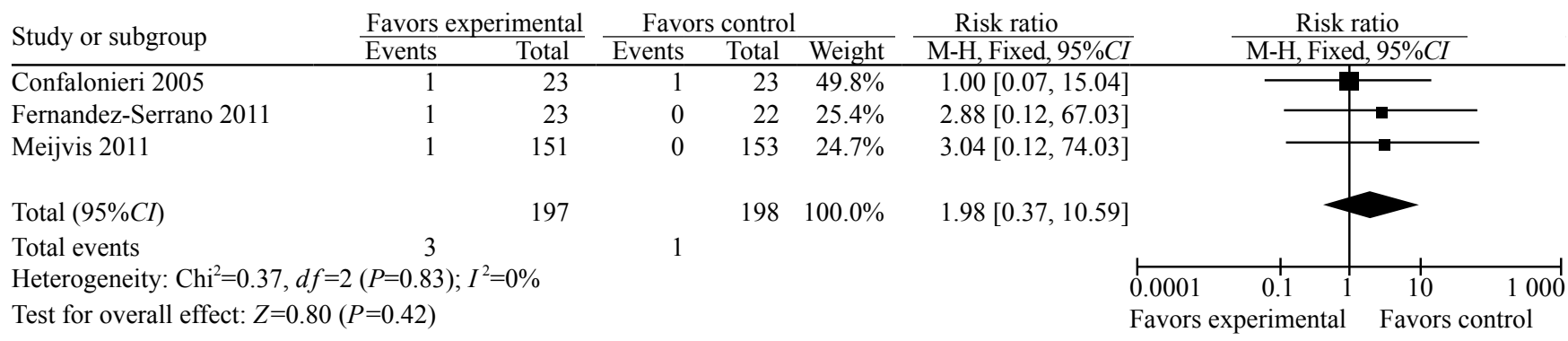

Figure 6. Comparison of the incidence of upper gastrointestinal bleeding in the two groups. 
no statistically significant difference $(Z=1.36, P=0.17)$ (Figure 6).

\section{DISCUSSION}

The inflammatory response of the host is a key factor determining the prognosis in CAP, which aims to destroy microorganism and control infection, but excessive inflammatory response is harmful to the host, resulting in early in-hospital mortality in patients with CAP. GCS as the most effective anti-inflammatory agents may be effective for severe CAP patients with adrenal insufficiency. Systemic GCS treatment is recommended in consensus guidelines of the Infectious Diseases Society of America/American Thoracic Society on the management of severe CAP in patients, but the evidence was from small sample sizes in RCTs.

A systematic review showed that administration of GCS in patients with severe CAP was associated with a lower mortality rate than those treated with placebo. ${ }^{[14]}$ The current meta-analysis demonstrated that GCS treatment decreased the mortality of CAP, but no significant difference was detected. GCS treatment could shorten the length of hospital stay (LOS), but didn't shorten the length of ICU stay of patients with severe CAP. This finding might be due to the insufficient information from primary publications and the accuracy of the contents. Therapeutic benefit and safety could be expected during the treatment of severe CAP with GCS; however, severe side effects such as super infection, hyperglycemia, gastroduodenal bleeding, and muscle weakness after prolonged GCS treatment may occur in patients with severe CAP. There was no statistically significant difference in adverse effect in patients treated with GCS compared with patients treated with standard methods, indicating that the safety of GCS treatment as an adjunctive therapy for CAP.

In this meta-analysis, 944 patients were hospitalized for CAP, including those with mild to severe CAP. Adrenal function was not assessed in most RCTs. In addition, the doses and duration of GCS treatment were different among the studies, which contributed to a significant clinical heterogeneity in systematic evaluation if no subgroup analysis was performed in the RCTs. Heterogeneity was found in pooled analysis on the length of hospital stay, the length of ICU stay and super infection with GCS treatment in RCTs. Therefore, sensitivity and subgroup analyses were performed again in this meta-analysis to assess the length of hospital stay, the length of ICU stay and super infection comparing
GCS with routine treatment of CAP patients. The results were consistent with those from the original studies, showing the stability of meta-analysis.

This meta-analysis has certain limitations. The scientific integrity of results and conclusions from meta-analyses could be influenced by the type of GCS, the dosage, and the treatment duration in each RCT. Further, the seven RCTs included in this meta-analysis have methodological differences: (1) four RCTs did not depict the concealment of randomization allocation; (2) only four RCTs were performed using doubleblinding methods, the remaining three were performed using a single-blind method; (3) the articles included in the analysis were limited to publications in English or Chinese only.

Hence, GCS as an adjunctive therapy is valuable for patients with CAP. When it is cautiously used in clinical setting, complications should be determined if necessary. Further RCTs with a large sample size, higher quality, and a long-term follow-up period are warranted to define the indication of GCS for patients with CAP and to evaluate the appropriate type of GCS, dosage, and the duration of treatment.

\section{Funding: None.}

Ethical approval: Not needed.

Conflicts of interest: The authors declare that there is no conflict of interest relevant to the content of the article.

Contributors: Chen LP proposed the study, analyzed the data and wrote the first draft. All authors contributed to the design and interpretation of the study and to further drafts.

\section{REFERENCES}

1 Sibila O, Agusti C, Torres A. Corticosteroids in severe pneumonia. Eur Respir J 2008; 32: 259-264.

2 Anderson RN, Smith BL. Deaths: leading causes for 2002. Natl Vital State Rep 2005; 53: 1-89.

3 Mandell LA, Wunderink RG, Anzueto A, Bartlett JG, Campbell GD, Dean NC, et al. Infectious Diseases Society of America/ American Thoracic Society consensus guidelines on the management of community-acquired pneumonia in adults. Clin Infect Dis 2007; 44: S27-S72.

4 Niederman MS. Recent advances in community-acquired pneumonia: inpatient and outpatient. Chest 2007; 131: 12051215.

5 Annane D, Maxime V, Ibrahim F, Alvarez JC, Abe E, Boudou P. Diagnosis of adrenal insufficiency in severe sepsis and septic shock. Am J Respir Crit Care Med 2006; 174: 1319-1326.

6 de Jong MF, Beishuizen A, Spijkstra JJ, Groeneveld AB. Relative adrenal insufficiency as a predictor of disease severity, mortality, and beneficial effects of corticosteroid treatment in septic shock. Crit Care Med 2007 ; 35: 1896-1903. 
7 Snijders D, Daniels JM, de Graaff CS, van der Werf TS, Boersma WG. Efficacy of corticosteroids in community-acquired pneumonia: a randomized double-blinded clinical trial. Am J Respir Crit Care Med 2010; 181: 975-982.

8 Remmelts HH, Meijvis SC, Heijligenberg R, Rijkers GT, Oosterheert JJ, Bos WJ, et al. Biomarkers define the clinical response to dexamethasone in community-acquired pneumonia. J Infect 2012; 65: 25-31.

9 Confalonieri M, Urbino R, Potena A, Piattella M, Parigi P, Puccio G, et al. Hydrocortisone infusion for severe communityacquired pneumonia: a preliminary randomized study. Am J Respir Crit Care Med 2005; 171: 242-248.

10 Mikami K, Suzuki M, Kitagawa H, Kawakami M, Hirota N, Yamaguchi H, et al. Efficacy of corticosteroids in the treatment of community-acquired pneumonia requiring hospitalization. Lung 2007; 185: 249-255.

11 Marik PE, Pastores SM, Annane D, Meduri GU, Sprung CL, Arlt $\mathrm{W}$, et al. Recommendations for the diagnosis and management of corticosteroid insufficiency in critically ill adult patients: consensus statements from an international task force by the American College of Critical Care Medicine. Crit Care Med 2008; 36: 1937-1949.

12 Meijvis SC, Hardeman H, Remmelts HH, Heijligenberg R, Rijkers GT, van Velzen-Blad H, et al. Dexamethasone and length of hospital stay in patients with community-acquired pneumonia: a randomised, double-blind, placebo-controlled trial. Lancet 2011; 377: 2023-2030.

13 Fernández-Serrano S, Dorca J, Garcia-Vidal C, FernándezSabé N, Carratalà J, Fernández-Agüera A, et al. Effect of corticosteroids on the clinical course of community-acquired pneumonia: a randomized controlled trial. Critical Care 2011; 15: R96.

14 Siempos II, Vardakas KZ, Kopterides P, Falagas ME. Adjunctive therapies for community-acquired pneumonia: a systematic review. J Antimicrob Chemother 2008; 62: 661-668.

Received January 21, 2015 Accepted after revision May 19, 2015 